\title{
Abstracts from BSM Conference 2010
}

\section{CLIMATECHANGEANDHUMANHEALTH Quazi Tarikul Islam}

World Health Organization (WHO) in 1993 defines "Environmental health comprises these aspects of human health including quality of life that are determined by physical, chemical, biologic, social and psychosocial factors in the environment

In 1969 the Apollo moon short provided extra ordinary photograph of this planet suspended in space. This transformed how we thought about the biosphere and its limit. Climate and weather has a powerful impact on human health and well being. Climate change is happening now and started as long as 100 year before producing Green House Gas (GHG) which ultimately causes warming of the climate system

The GHG are -Carbon dioxide which is produced from fossil fuel combustion and wildfire and remain in the environment $100 \mathrm{yrs}$, Methane which is produced from wetland \& moulds remain in the environment for $12 \mathrm{yrs}$, Halo carbon produced from industries and remain the environment 200 yrs, Per fluro carbon produced from electric devices remain in the environment for $5000 \mathrm{yrs}$.

Though USA. And China are the countries of the World producing major GHG emission but the sufferer are the developing countries, of them Bangladesh is mostly affected. In last 30 years (1970-2000) consumption of fuel increases double. $60 \%$ of Deforestation occurs in last 100 years. The surface temperature of earth increase from 1.8 - 4 degree centigrade which will cause sea level water to increase from $18-50$ inches by the year 2050. So 21st century is a great threat for human health:

(a) Flood : Water bone disease, drowning, snake bite. (b).Earth quake: Gross disaster and loss of life,(c).Draught: Water scarcity, heat wave, (d).Cyclone: loss of lives(e).Air pollution: Excess automobile and emission of carbon dioxide and other gases cause Br. Asthma, COPD and heart diseases. (f).Water borne disease: Lack of safe water, sea water contain plankton which is a reservoir of V. cholerae,(g)Change in vectors of infections diseases Malaria, Dengue, Plague.

What we should do :a) Mitigation: By reducing GHG emission: Reducing energy usage and reduce, reuse, recycle at individual level \& national level and b)Adaptation: Prepare for impact of climate change

Bangladesh does not have any consolidated data how much we have been affected due to climate change on health.We need to go for lot of research. Regarding disaster management \& preparedness we are in much better position that any other country because we are use to. Let us reduces the globe temp at least 2 degree centigrade so that we can preserve our beautiful world more beautiful.

\section{GETTING BLOOD PRESSURE (BP)TO GOAL.}

\section{Virginia Hood}

Hypertension affects over one billion people worldwide and the prevalence is increasing at an alarming rate. The rates of high BP in US and in Bangladesh increase with age and are $50-70 \%$ of those aged greater than 60 years. High BP is a major risk factor for cardiovascular (CV) morbidity and mortality. For every $20 \mathrm{mmHg}$ rise in systolic BP over 115 $\mathrm{mmHg}$ the risk of adverse $\mathrm{CV}$ outcomes doubles. Reducing $\mathrm{BP}$ is the most effective modifying factor for reducing stroke, progression of kidney disease and cardiovascular disease. High BP results from a combination of genetic and environmental influences that cause volume and pressor dependent changes in the arterioles. Treatment goals are BP $<$ 129/79 mmHg for persons with diabetes, chronic kidney disease and cardiovascular disease and $<139 / 89$ for those without these conditions. Achieving these goals usually requires both lifestyle changes and medication use. Lifestyle changes including reducing sodium intake, stopping tobacco use, increasing physical activity, and eating heart healthy diets can reduce BP by 5-15 mmHg. Medications are best used in combinations so as to reduce volume and pressor effects. In those with difficult to control BP, combinations of a diuretic, ACE inhibitor or ARB, beta blocking or alpha plus beta blocking agent and a calcium channel blocking agent are usually required to get effective control and with best patient acceptance. Management of high BP requires setting goals and developing strategies for individuals and groups that require physician, patient and system participation.

\section{PITFALLINTHE DIAGNOSISAND MANAGEMENT OF TYPHOIDFEVER}

\section{Md.Amir Hossain, Shahena Akter}

Typhoid fever is an endemic disease many developing countries and sporadic in developed countries. Reliable data from which to estimate the disease burden in developing countries are difficult to obtain, since many hospitals lack facilities for blood culture for confirmation of diagnosis of 
the disease and up to 90 percent of patients with tyhoid are treated as outpatients. Community based studies have consistently shown higher levels of typhoid than public heath figures suggest.

Despite many new developments, the diagnosis of typhoid in much of the developing world is made on clinical criteria. This poses problems, since typhoid fever may mimic many common febrile illnesses without localizing signs. The early stages of enteric fever may be confused with conditions of many infectious diseases in tropics like malaria, brucellosis, dengue. etc. Recent data from South Asia indicate that the presentation of typhoid may be more dramatic in children younger than 5 years, with higher rates of complications and hospitalization. Multidrug resistant typhoid and paratyphoid infections are more severe with higher rates of toxicity, complications, and mortality than infections with sensitive strains. The mainstay of laboratory confirmation of diagnosis of typhoid fever is organism in blood culture which is only 40-60\% positive in early course of the disease. Although bone marrow cultures are more sensitive, they are difficult to obtain, relatively invasive, and of little use in public health settings. Classic Widal test lacks sensitivity and specificity. Typhidot or Tubex, have not proved to be sufficiently robust in large scale evaluations in community settings. A nested polymerase chain reaction using H1-d primers is a promising means of making a rapid diagnosis. The emergence of multidrug resistant typhoid in the 1990s led to widespread use of fluoroquinolones as the treatment of choice for suspected typhoid, especially in South Asia and South East Asia where the disease was endemic. In recent years, however, the emergence of resistance to quinolones has placed tremendous pressure on public health systems in developing countries as treatment options are limited. The World Health Organization's recommendations for multidrug resistant typhoid have shown that fluoroquinolones can achieve satisfactory cure rates, but parenteral ceftriaxone was associated with higher rates of relapse. Addressing this issue are indication of development of simple algorithms for diagnosis and management of typhoid fever in endemic areas.

\section{ANAPPROACHTO PATIENTS WITHACUTE FEBRILE CONFUSIONALSTATE}

\section{MdAzizul Kahhar}

Acute febrile confusional states are characterized by an acute change of cognition and a disturbance on consciousness, usually resulting from an underlying febrile medical condition or from medication or drug withdrawal. Although it is associated with increased mortality and morbidity, it is often not recognized by physicians. It is especially common in elderly patients and posses a substantial challenge for clinicians.

Delirium affects 10 to 30 percent of hospitalized patients with medical illness and most of them are febrile; more than 50 percent of high risk groups had a precipitating factor like infections to become confuse in an acute setting. The confused patients may comprises a wide range of symptoms like agitation, samnolence, withdrawl and psychosis. This variation of presentation can lead to diagnostic confusion and in some cases attribution of symptoms to a primary psychiatric disorder. Diagnosis can be improved by clinicians becoming more aware of hypoactive presentation, identify hyperactive and mixed presentation, incorporating cognitive assessment into routine practice and using simple screening instruments.

Febrile confused patients have different aetiology in different age groups. The intracranial aetiology should be sought immediately for early intervention, as mortality and morbidity will be increased if the approach to these groups becomes late. The extracranial causes should also be sought systematically, so that unnecessary investigation and delay can be prevented. Primary care physicians must be able to recognize the acute febrile confessional states so that the underlying aetiology can be ascertained and addressed. The management of acute febrile confusional states involves identifying and correcting the underlying problem, and symptomatically managing any behavioral or psychiatric symptoms. Low doses of antipsychotic drugs can help to control agitaton but benzodiazepines are indicated in specific situations. Environmental interventions, including frequent reorientation of patients by nursing stuff and education of patients and families should be employed in all cases.

\section{INFECTIONINCRITICAL CARE SETTING}

\section{A R M Saifuddin Ekram}

Critical care patients are at risk for developing endogenous and exogenous nosocomial infections. The compromised patient with his health at its low is subjected to extra risk of infection, which can be transmitted from his caregiver or his visitors. So high level of care is needed and self-hygiene should be at highest ebb. There should be monitoring of the infection pattern and the bacteria monitored in order to take the necessary steps to treat infection, prevent resistance development and preventing hospital acquired cross infection. Essential components of an infection-control 
program in the critical care environment are the structural design of the critical care unit and the surveillance, prevention, and control of infection functions. Management strategies include employee infection control and education. Knowledge and education are the most important management tools in the control of infection in the critical care environment. There are eight infection control indicators in hospitals in critical care setting. The indicator set focuses on: surgical wound infections, postoperative pneumonia, use of urinary catheterization, ventilator-associated pneumonia, primary bloodstream infection, endometritis, adequacy of surveillance, and employee measles immunization. Critical care unit staff members may be involved in using indicator rates. Following the trend rates should suggest organizational systems and processes of care in critical care settings that may warrant further examination and improvement.

\section{RECENTADVANCESINTHETREATMENTOFMALARIA}

\section{NJ White}

Major developments in recent years in the treatment of malaria are the introduction of the plant derived artemisinins from China, and the acceptance that combinations should be used to ensure high cure rates and prevent the emergence of antimalarial drug resistance. In the hospital management of severe malaria parenteral artesunate was shown to reduce the mortality of severe malaria by $35 \%$ compared with quinine in a very large multicentre trial. No adjuvant treatments have proved effective to date. Community based preferral treatment with rectal artesunate of suspected severe malaria in children reduced mortality 25\%. In uncomplicated falciparum malaria artemisinin based combination treatments (ACTs) have become the first-line of management. Where deployed extensively they have contributed to a significant decline in malaria. Artemether-lumefantrine, artesunatemefloquine, and dihydroartemisinin-piperaquine are highly effective everywhere, and well tolerated. Fixed dose combinations improve adherence and tolerability. Dose optimization in young children and pregnant women remains an urgent priority. There has been less progress in the management of vivax malaria. Chloroquine and primaquine remain the drugs of choice although there is increasing evidence of chloroquine reistance. ACTs are also highly effective. Optimising the use of primaquine, sixty years after its introduction, still remains a research priority.

\section{EXAMINATION OFAPATIENTWITHHYPERTENSION}

\section{Md. Enamul Karim}

Hypertension is a very common clinical problem. The relationship between blood pressure and risk of cardiovascular disease is linear, continuous, and independent of other risk factors. For people of 40 to 70 years old, each increment of either $20 \mathrm{~mm} \mathrm{Hg}$ in systolic blood pressure or $10 \mathrm{~mm} \mathrm{Hg}$ in diastolic blood pressure doubles the risk of cardiovascular disease. If the patient smokes or has elevated cholesterol, other cardiovascular risk factors, or the metabolic syndrome, the risk is even higher. Thereby it is very much important to examine hypertensive patients accurately and treat accordingly. For most patients, accurate blood pressure measurement, a focused history and physical examination, and a handful of basic tests are enough. The goal of the hypertension evaluation is to find answer of four questions: Does the patient have sustained hypertension? And if so is the hypertension primary or secondary? Does the patient have other cardiovascular risk factors? Does he or she have evidence of target organ damage? The usual goal of antihypertensive treatment is less than 140/ $90 \mathrm{~mm}$ Hg. However, the target is lower, less than 130/80 mm Hg, for those with diabetes or target organ damage such as heart failure or renal disease. Thus, it is important to detect these conditions in the evaluation of the hypertensive patient. Also a routine evaluation of hypertensive patients is mandatory; which include at a minimum: a hemoglobin or hematocrit measurement, urinalysis with microscopic examination, serum electrolyte concentrations, serum creatinine concentrations, serum glucose concentration, fasting lipid profile and 12-lead electrocardiogram.

\section{POISONING AMONG COMMUTERS IN DHAKA, BANGLADESH: PROSPECTIVE CLINICALSTUDYAND TOXICOLOGICAL SCREENING}

Ariful Basher, Stefan W. Toennes, Rashed Chowdhury, Syed M.Arif, M.A. Kashem Khandaker, Ulrich Kuch, M.Abul Faiz

Objective: Drug-facilitated robberies primarily of public transport passengers are a massive and still increasing public health and law enforcement challenge in Dhaka This study aimed at identifying the poisoning agents used and estimating their dosages.

Methods: Prospective clinical and toxicological screening using LC-TOF/MS and LC-MS/MS analysis of blood and urine samples from 38 suspected poisoning victims admitted unconscious at Dhaka Medical College Hospital between October 2008 and January 2009. Blood samples were obtained 
on admission and one hour later to identify increasing concentrations that would indicate a continuing absorption phase.

Results: All patients were male, 17-60 years old, could not estimate the time between substance ingestion and loss of consciousness, and had lost valuables. Fifty percent had a GCS of 5-10. Most patients were poisoned waiting at a bus stand or on close-by markets (63\%), and on buses (29\%); they had taken tea (21\%), cold drinks (16\%), pickles (15\%), herbal medication (2\%) or smoked a cigarette (5\%). LC-TOF/ MS screening (22 cases) and LC-MS/MS lorazepam target analysis (4 cases) revealed pharmacologically active concentrations of lorazepam in blood samples of all 26 cases (217’\%132 a\%g/l, mean’\%SD), of midazolam in 12 (149\%\%99 $\mathrm{a} \% \mathrm{~g} / \mathrm{l})$, of diazepam in 3 (217\%\%144 a\%g/l) and of nordiazepam in 6 cases (364\%\%186 a\%g/l). In 5 of the 26 cases the lorazepam concentration of the second blood sample was at least $15 \%$ higher indicating continuing absorption. This suggests that only these 5 patients were admitted within the 1-2 hour-long absorption phase. Minimum ingested doses, estimated using the lower boundary of the benzodiazepines' volumes of distribution and the upper boundary of the bioavailabilities, were 11 '\%7 mg (mean’\%SD) lorazepam, 13’\%9 mg diazepam, 20’\%10 mg nordiazepam and 15\% $10 \mathrm{mg}$ midazolam. Three patients were hypotensive; 2 of these had lorazepam levels $>250 \mathrm{ng} / \mathrm{ml}$ and a GCS of 5-10. Almost all of the patients left the hospital unnoticed after clearing up, two likely prematurely within 12 hours of admission, and no mortality was observed.

Conclusion: This study reveals flexibility of the criminals in using different mixtures of benzodiazepines (in our earlier study a single drug, lorazepam, was common). They show that an up to 12-fold overdose of benzodiazepines did not lead to life-threatening symptoms and have important implications for the clinical management of these drugfacilitated crimes. In particular, they highlight the urgent need for rapid diagnostic and analytical forensic and clinical toxicology facilities, modifications to emergency department protocols, and post-recovery psychological support for victims

\section{ACUTE PHASE PROTEINS IN STROKE PATIENTS INA TERTIARY CARE HOSPITAL}

Ekramul Mustafa, Mohammad Aftab Haleem, Md. Mahmudur Rahman Siddiqui, Jabed Iqbal, Ahmed Hossain, Quazi Tarikul Islam

Objectives: The onset of cerebral ischemia triggers a cascade of proinflammatory molecular and cellular events. Clinical studies suggest that the strength of this acute response is important in early and late clinical outcomes. A large number of proteins (i.e., fibrinogen, $\mathrm{C}$ - reactive protein (CRP), complement 3 (C 3), complement 4 (C 4), Tumor necrosis factor (TNF), and haptoglobulin (Hp) have considered as Acute Phase Proteins (APPs). However, Fibrinogen \& CRP have gained wide popularity for monitoring inflammation in many different disease states. Thus the aim of this study is to determine the correlation between acute ischemic stroke and the acute phase reactants (CRP, ESR).

Materials and Methods: It was a prospective descriptive study. The acute stroke patients admitted in one of the medicine unit of Dhaka Medical College Hospital(DMCH) were enrolled. The study group consists of 30 subjects (20 male and 10 female patients). 15 patients of ischemic stroke and 15 patients of haemorrhagic patients were included in the study. Plasma levels of two APPs (CRP \& ESR) were measured within 72 hours after the onset of stroke. In addition several other risk factors were also determined

Results: The mean age of subjects was (60 '\%5). Both CRP and ESR in acute ischemic stroke was found higher and statistically significant than hemorrhagic stroke $(p=0.003$, $\mathrm{p}=0.02$ )

Conclusion: Elevation of CRP \& ESR immediately after ischemic stroke is an important diagnostic parameter and is not a good parameter for haemorrhagic stroke.

\section{PATTERN AND ETIOLOGY OF ELECTROLYTE IMBALANCE INHOSPITALIZED DIABETIC PATIENTS- ASTUDY OF 100 PATIENTS}

\section{Ahmed JU, Hauque HF, Hossain D, Musa AKM, Uddin KN}

Objective: To see the pattern and etiology of electrolyte imbalance in hospitalized diabetic patients.

Materials and Methods: It was a cross sectional study, carried out from January to December 2008 in BIRDEM Hospital among 100 hospitalized diabetic patients. All participants were interviewed using a structured questionnaire, which covered socio-demographic data, life style and food habit. Anthropometrical measurement, BP, Serum electrolytes were measured in standardized way.

Results: Among the enrolled patients, 74 (74\%) were male and 26 (26\%) were female The mean age of the patients was 60.18 \% 12.15 (Range 38-90). Among the patients, the mean of history of DM was 10.96 years with SD '\% 8.05. The mean Blood urea nitrogen (BUN) was 53.35 with SD \% $\%$ 33.23. The mean Serum creatinine (S. creatinine) was $1.70 \mathrm{mg} / \mathrm{dl}$ with SD \% 0.94 . 
Regarding treatment, 83 (83\%) of them were on insulin therapy, 22 (22\%) of them were on oral hypoglycemic agents, $6(6 \%)$ of them were on diet and exercise. In regard of co morbid condition, 61 (61\%) of them had Hypertension (HTN), 24 (24\%) of them had Ischemic Heart diseases (IHD), 21 (21\%) of them had Renal diseases, 8 (8\%) of them had Dyslipidemia and 4 (4\%) of them had Liver diseases. Regarding the complication of diabetes, 41 (41\%) of them had Diabetic Nephropathy, 13 (13\%) of them had Diabetic Neuropathy and 12 (12\%) of them had Diabetic Retinopathy. Regarding electrolyte imbalance, 80 (80\%) of them had Hyponatraemia, 36 (36\%) of them had Hypokalaemia, 14 (14\%) of them had Hyperkalaemia, and 38 (38\%) of them had Hypomagnesaemia. In regards of causes of electrolyte imbalance, 51 (51\%) of them had vomiting, 5 (5\%) of them had diarrhea, 28 (28\%) of them were having various drugs, 24 (24\%) of them had Renal failure, 2 (2\%) of them had other causes and 4 (4\%) of them had unknown causes.

Conclusion: Electrolyte imbalance is a common problem encountered in diabetic patients. Diarrhea and vomiting are a common cause, often precipitating on pre-existing renal disease.

\section{BASELINE SURVEY OF CARDIOVASCULARDISEASES RISK FACTORS IN A RURAL POPULATION OF BANGLADESH}

Ridwanur Rahman, Md Mujibur Rahman, Rubina Yasmin, A N M Momenuzzaman, Jawhar lal Sen, Sahjada Selim, Saifullah Khan

Objective: The prevalence of cardiovascular disease (CVD) has been increasing in Bangladesh. To study the prevalence of risk factors of cardiovascular diseases among a geographically defined rural population of Bangladesh aged 25 years.

Material and Methods:Study design: Descriptive cross sectional survey.

Setting: Sanora Union of Dhamrai Upazilla, Bangladesh.

Participants: 9675(male and female) from 4227 households, a total of 5332 individuals data were collected.

Main outcome measures: social and economic circumstances, self reported symptoms and diseases, anthropometric measurement and blood pressure measurement.

Results: Social and economic circumstances of people of that area are similar to national data. Illiteracy rate $62.6 \%$ in female and $40.2 \%$ in male. $48 \%$ male were involved in agriculture and 93.7\% female were house wife. 58.1\% family had 4-6 members. 70\% families were belonged to lower and low middle class. $12.7 \%$ population had family history of CVD, among them stroke were present in 40\%. 39.9\% female and $17.1 \%$ male had sedentary physical activity. Prevalence of smoking was $42.1 \%$ in male and $1 \%$ in female, and smokeless tobaccos were used by $34.6 \%$ male and $41.4 \%$ female. $10.3 \%$ male and $14.1 \%$ female had BMI $>25$. Adverse waist Hip ratio ( $>1$ ) were present in $2.2 \%$ male and $9.2 \%$ in female. 5.3\% male and 7.3\% female were hypertensive. $2 \%$ male and $1.7 \%$ female were diabetic.

Conclusion: Prevalence of cardiovascular disease risk factors are higher in rural area. Prevention, detection and treatment of CVD risk factors should be an important component of our national strategy to reduce the increasing burden of cardiovascular disease in Bangladesh.

\section{SPUTUMCULTURE \& DRUGSENSITIVITYPATTERNOF COMMUNITYACQUIRED PNEUMONIA IN DIABETIC PATIENTS AND THEIR CORRELATION WITH GLYCAEMIC STATUS}

Jamal Uddin Ahmed, Tanvir Ahmed, Md. Delwar Hossain, AKM Musa, Khwaja Nazim Uddin

Introduction: Diabetes Mellitus is an immunosuppressive state leading to increased susceptibility to various infections including pneumonia. Pneumonia in diabetic patients is often atypical, caused by more virulent organisms and associated with increased antibiotic resistance.

Materials and Methods: To justify this aspect, a crosssectional study was conducted in the Department of Internal Medicine \& Pulmonology of BIRDEM from January 2008 to December 2009.

Results: A total of 122 hospitalized diabetic patients with community acquired pneumonia were taken in the study. Of them 82 (67\%) were male, 40 (33\%) were female. Mean age of the patients was - $60.79 \% 11.5$ years. Mean duration of diabetes was - 2.33 '\%1.2 years. Sputum for culture shows that out of 122 (100\%) patients, Klebsiella pneumonia was detected in 54 (44.3\%) patients, Pseudomonas in 16 (13.1\%) patients, E.coli in 10 (8.2\%) patients, Staphylococcus aureus in 18 (14.8\%), Acinetobacter in 10 (8.2) and 14 (12\%) patients had growth of other organisms. Sputum pus cell was significant in 64 (52.5\%) samples. Antibiotic sensitivity pattern shows that among 54 (100\%) growth of Klebsiella, Ceftriaxone was 10 (18.5\%) sensitive vs 44 (81.5\%) resistant, Ceftazidime was 14 (25.9\%) sensitive vs 40 (74.1\%) resistant [p= .04], Amikacin was 40 (74.1\%) sensitive vs 14 (25.9\%) 
resistant [ $\mathrm{p}=.035]$, Imipenem was 52 (96.3\%) sensitive vs 2 (3.7\%) resistant $[\mathrm{p}=.000]$, Ciprofloxacin was 26 (48.1\%) sensitive vs 28 (51.9\%) resistant. Mean HbA1c was - 8.6 '\%1.89. Correlation with growth of organism shows that 52 (96.3\%) of Klebsiella growth occurred with HbA1c $>7$.

Conclusion: Community acquired pneumonia in uncontrolled diabetic patients are more frequently due to Klebsiella, Pseudomonas and S. aureus and frequently they are resistant to Ceftriaxone and oral antibiotics. So, empirical treatment of diabetic pneumonia with Ceftriaxone or oral antibiotic is not a good option and alternative antibiotics should be used.

\section{GLYCEMIC STATUS DURING ACUTE CORONARY SYNDROME OF NON-DIABETIC PATIENTS}

\section{Md. Zillur Rahman,ARM Saifuddin Ekram,Quazi Tarikul Islam, Md. Mahmudur Rahman Siddiqui}

Objectives: To measure glycaemic status during acute coronary syndrome of previously non-diabetic patients that will give the information about the frequency of acute hyperglycemia in acute coronary syndrome (ACS) among Bangladeshi population and it will help us in future to take appropriate measure for hyperglycemia related ACS complications.

Materials and Methods: This is an observational cross sectional study performed in Rajshahi Medical College Hospital. A total of 725 patients with ACS got admitted in study period. Among them, 248 (216 male \& 32 female) were selected as non-diabetic by proper history taking, documentary papers \& HbA1c estimation.HbA1c more than 7 were ruled out from the study.The enrolled patients information were recorded in a preformed structured Case Record Form(CRF). A fasting and 2 hrs after 75 gm glucose (OGTT) were performed within 72 hrs of admission.

Results: Out of 248 study population, 135 (54.44\%) had glucose abnormalities. Male was 87.10\% (216). Among male, IGT (pre-diabetic) was 31.94\% and diabetic 24.54\% (53) which was much more than that of female. Normal glucose tolerance (NGT) was found in 45.6\% (113) cases, IGT 30.6\% (76) and diabetic were $23.8 \%$ (59) cases. Between $41-50$ years the frequency of diabetes was more common $24.71 \%$ \& IGT was more common (34.12\%) in the age group of 31-40 years. Among our study population, smokers were 69.76\% (173) and their glucose abnormalities were $56.64 \%$ including prediabetic $77.63 \%$ and diabetics $66.10 \%$ (39). Glucose abnormalities were observed with frequency among active and younger groups (41-50) years, smoker \& hypertensive than that of extreme age groups, normotensive and nonsmokers.
Conclusion: We should create awareness about a new risk factor- acute hyperglycemia during ACS and take appropriate and effective measures to reduce morbidity as well as mortality as a consequence of acute hyperglycemia during ACS with or without diabetes.

\section{AETIOLOGICAL PATTERN, BIOCHEMICAL EVALUATION AND OUTCOME OF ACUTE VIRAL HEPATITISINPREGNANCY}

\section{Fazle Rabbi Mohammed, H.A.M. Nazmul Ahasan}

Objective: Greater mortality and morbidity was detected in acute viral hepatitis (AVH) during pregnancy. Acute hepatic failure (AHF) is particularly common in pregnancy with hepatitis $E$ through out the world with a fatality rate up to $20 \%$. This rate is higher in developing communities. Hepatitis $\mathrm{B}$ is rising alarmingly in all groups of people. This study aimed to find out the serological pattern, complications and outcome of AVH in pregnant and non pregnant cases.

Methods: This open labeled observational study was executed in Dhaka Medical College Hospital from September, 2008 to February, 2009 and 50 patients having clinical manifestation of acute viral hepatitis, confirmed by viral markers, were recruited.

Results: Among 50 cases, 13 were pregnant and remaining 37 included both male and non pregnant female with a ratio $1: 2.85$. The majority of pregnant cases (62\%) was in second trimester and $38 \%$ was in third trimester. Hepatitis E was present in $85 \%$ pregnant cases and 35\% non pregnant and the rate was $15 \%$ and $47 \%$ respectively in case of hepatitis B. Hepatitis A was not detected in pregnancy but it was $18 \%$ in non pregnant group. Compare to pregnant and non pregnant patients, the mean value of serum bilirubin was $10.77 \mathrm{mg} / \mathrm{dl}$ and $10.12 \mathrm{mg} / \mathrm{dl}$, the mean value of serum alanine aminotransferase was $760.54 \mathrm{U} / \mathrm{l}$ and $610.14 \mathrm{U} / \mathrm{l}$ and the mean value of prothrombin time was 28.98 second and 20.44 second. AHF was detected in 54\% pregnant; all of them had hepatitis $\mathrm{E}$ and it was $13 \%$ among non pregnant of whom $60 \%$ had hepatitis E and remaining $40 \%$ had hepatitis B. Death rate was $46 \%$ among pregnant cases and all of them had hepatitis $\mathrm{E}$ and it was 8\% in non pregnant where hepatitis $\mathrm{E}$ and $\mathrm{B}$ was $67 \%$ and $33 \%$ respectively.

Conclusion: We should give special emphasis on pregnancy with Hepatitis E to reduce the rate of maternal mortality. Life style modification, like proper sanitation facilities or availability of pure drinking water, can be achieved by mass population awareness. Vaccination programme should be initiated effectively. These initiatives may reduce the incidence of hepatitis in coming future. 


\section{CAROTID ARTERY PLAQUE AND LIPID PROFILE IN ISCHAEMIC STROKE PATIENTS}

Md. Nurul Islam, A R M Saifuddin Ekram, Md. QuamaruddinAhmed, Md.Azizul Haque, AhmadAli, Md. Mahbubur Rahman Khan, Quazi Tarikul Islam

Objective: To observe the frequency of carotid artery plaque in ischaemic stroke patients and its correlation with dyslipidaemia.

Materials and Methods: This observational hospital based study was conducted at the Department of Medicine, Rajshahi Medical College Hospital on fifty (50) patients of stroke with cerebral infarction confirmed by CT scan of the brain.

Results: Hypertension was the commonest risk factor (74\%), followed by smoking (68\%), carotid artery stenosis (58\%), dyslipidaemia (48\%), diabetes mellitus (42\%), family history of stroke (34\%), obesity (22\%), male gender were predominant than female (80\% vs. 20\%) and mean age ('\% SD) was $61.72 \% 11.31$ years. The frequency of significant carotid artery plaque in acute ischaemic stroke patient was on right side $52 \%$, on left side $40 \%$ and on both side $34 \%$. Left internal carotid artery showed minor positive correlation with serum cholesterol, LDL cholesterol and HDL cholesterol ( $r=0.081, p=0.57)$ and Right internal carotid artery showed similar minor positive correlation with LDL and HDL cholesterol ( $\mathrm{r}=-0.038$, $\mathrm{p}=0.79$ ) but both arterial plaque had insignificant association with dyslipidaemia.

Conclusion: Results indicate that in the common carotid as well as in the internal and external carotid arteries intima media thickness are a good predictor of ischaemic stroke. Though this thickness has less significant correlation with the lipid levels in blood, but this non invasive method can be used successfully to identify the high risk patients prone to develop stroke due to carotid plaque formation and increased intima media thickness.

\section{STUDY OF BODY MASS INDEX (BMI) IN ADULT HYPERTENSIVE PATIENTS IN RANGPUR, BANGLADESH}

MA Haque, F Nasreen, BB Das, SMS Jahan, M M Rahman. MLAli, MZ Hossain, LA Khan, NA Siddiq

Objective: Despite a growing burden of obesity and hypertension in developing countries, there is limited information on the contribution of body mass index (BMI) to blood pressure(BP). The purpose of this study was to examine the association of BMI in hypertensive patients in a district (Rangpur), Bangladesh.
Materials and Methods: Study place: Hypeertension and research centre,Rangpur,Bangladesh, Study period: Dec,09 to Jan,2010, Study design: Cross- sectional descriptive study, Population: 435 random;y selected hypertensive patinets

Data Collection: Data for tis study were generated in apublic gathering.Export fair, 2009, Rangpur, Bangladesh in apreformed questionnares.Sociodemographic data such as age, sex, occupation,monthly income and Body Mass Index (BMI) and blood pressure were recorded by trined persons and doctors.

Inclusion criteria: Adult hypertensive patients in between the age 20-59yrs

Exclusion criteria: Childen and adolscent below 20 year, and pregnancy and gross physical abnormality against the physical measurements were the exclusion criteria.

Operational definition: BMI was calculated as weight in $\mathrm{Kg}$ over Height in meters squared. Normal BMI defined as BMI in 18.5 to $24.9 \mathrm{~kg} / \mathrm{m} 2$, overweight as BMI $25-29.9 \mathrm{~kg} / \mathrm{m} 2$ or obese as BMI > 30kg/m2. Hypertensive patients were grouped into stage-1(defined as SBP 140-159 mm of Hg and DBP 90-99 mm Hg) and Stage -2(defined as SBP $160 \mathrm{~mm}$ of $\mathrm{Hg}$ and DBP $100 \mathrm{~mm}$ hg)

Statistical analysis: Windows SPSS-version 12

Results: A total of 452 hypertensive patients of 20-59 years aged were analyzed. Among the study group majority 52.9\% (n-230) had stage -1 hypertension and 47.1\% (n-205) had stage-2 hypertension. The mean age of the population was 37.98 years and most of the population (59.3\%) was in the age group of $30-39$ years.In The study group $49.56 \%$ of stage -1 and 605 of stage -2 hypertensive patients were overweight or obese 9BMI > $25 \mathrm{~kg} / \mathrm{m} 2)$. The study showed there was significant association between hypertension and BMI ( u2287.970,df=3,p-0.000) Regression analysis showed that change of hypertension on BMI was .45 with Beta coefficients of 0.064 . The risk of developing hypertension in overweight or obese group of population is more $(\mathrm{OR}=1.52)$

Conclusion: BMI is significantly associated with hypertension and increase the risk of hypertension in young adult group.

\section{PRESENTATION AND PATTERN OF ORGAN INVOLVEMENT IN SLE - AN EXPERIENCE IN A TERTIARY CARE HOSPITAL INBANGLADESH}

MM Rahman, SGM Mawla, S Jahan, MN Hasan, SMEAli,A Rahim, M Rahman, AA Imran, KMosharaf

Objective: The objective of this study was to find out the pattern of clinical presentations, to see the type of organ 
involvement and SLE disease activity index (SLEDAI) on admission.

Materials \& Methods: This retrospective study included 37 patients of SLE, diagnosed on the basis of ARA criteria. All patients' information was recorded in a preformed data sheet and data were analyzed manually.

Results: Among 37 patients, 33 (89.1\%) were female and 4 (10.8\%) were male. Female: Male ratio was 8.9. Age of the patients was ranging from 13 to 57 years. Most of the patients, 32 (86.5\%), were between the ages of 13-40 years. The most common initial manifestations were fever 29 (78.37\%), joint pain 28 (75\%), oral ulceration 19 (51\%), alopecia 14 (37.83\%) and photosensitivity 10 (27\%). Organs commonly involved were renal 11 (29.72\%), neurologic 6 (16.21\%), hematologic (anemia, bleeding and leucopenia) 13 (35.13\%), cardiac 6 (16.21\%), pulmonary 7 (18.91\%), and cutaneous 24 (64.86\%). Antinuclear antibodies, anti-double stranded DNA antibodies, and low C3 or C4 level were retrieved in 30 (81\%), 23 (62.16\%), and 8 (2162\%) of patients respectively. Renal Biopsy was indicated and done in 11 cases. The results reflected no cases in class-I, class-II and class-VI and 1 (9\%) in class-III, 7 (63.66\%) in class-IV and 2 (18\%) in class-V histopathological study. No glomeruli were found 1 case. SLEDAI was calculated in 27 patients and it was ranging from 6 to 58 .

Conclusion: Initial manifestations of SLE are diverse and often severe. The diagnosis of SLE should be promptly considered in any patients with prolonged febrile illness in young adult female with multi organ involvement.

\section{FROMTRIALSTO PRACTICE}

\section{Thomas Kjellstr $\div \mathrm{m}$}

The lecture will cover the planning process of a trial and the critical moment of a successful trial as well as illustrating the organization of a clinical trial. The quality of a trial is determined by the relevance of the design, the quality of the protocol, the quality of the performance and the quality of the database.

Also the factors affecting selection of patients for treatment will be discussed.

A more extensive discussion will be spent on evidence based medicine, and the criteria's for implementing study reports into clinical practice. The problems of implementing new knowledge into practise will be addressed as well as the different barriers for implementing guidelines.

Examples of clinical studies will be discussed from the clinical point of view.
The aim of the lecture is to help understanding how and which scientific reports and trials could be included in the daily clinical practise.

\section{NON-COMMUNICABLE DISEASES (NCD) AND EMERGINGTHREAT}

\section{Md. Ridwanur Rahman}

The presentation describes the NCDs as emerging threat. Non-communicable diseases are defined as a set of chronic diseases of major public health importance, such as cardiovascular diseases, cancer, diabetes mellitus, lung diseases such as asthma and chronic obstructive pulmonary disease, the development of which is influenced by one or more common risk factors (e.g., smoking, diets rich in energy and fat, physical inactivity and stress). The literary meaning that diseases which are not communicable (infectious) are non-communicable diseases is also true. But, the diseases of major public health importance, which are considered eminently preventable and/or controllable, are the diseases included under the term NCDs.

The world is clearly witnessing a growing man-made epidemic of NCDs which is being greatly aggravated by a rapidly ageing global population. It has been estimated that by the year 2020 up to three-quarters of all deaths in the world will result from NCDs, and Ischaemic Heart Disease and Depression will top the list of major public health problems in the world, and that there will be a significant increase in the disease burden from lung cancer. In 2002, non communicable chronic diseases were the leading cause of DALYs in all regions of the world except for Sub Saharan Africa. NCDs accounted for 47\% DALYs and 53\% worldwide deaths in 2002 and the prevalence of NCDs is projected to increase considerably over the next 20 years.

There is considerable diversity in the distribution of NCDs both between countries and regions of the world as well as differences within countries. The rise of NCDs in developing countries is inextricably linked to economic and cultural globalisation. Much of the projected rise in NCDs is preventable, particularly that due to smoking, poor diet, physical inactivity and obesity. Health systems in developing countries face both a growing need for prevention programmes and increasing numbers of individuals requiring treatment.

A public heath approach which focuses on population and risk factors rather than on individuals' symptoms or diseases is important to achieve the goal of promoting health and preventing diseases, addressing the underlying factors that determine health, and increasing the effectiveness and efficiency of healthcare system. 
Bangladesh has one of the most vulnerable economies, characterized by extremely high population density, low resource base, and high incidence of natural disasters. There is never as much funding as is needed to address all important health problems, so priorities need to be set. Priority setting is imperative for the rational utilisation of resources for public health programmes in a community. However, identification of priority health areas is not easy. Whether or not a particular disease or health condition should be focused and targeted for preventive activities depends on a number of factors.

Over the past two decades, some developed countries have gone through the process of identifying health priorities and started working on the identified health priority areas. For example, Australia has selected seven National Health Priority Areas for action, including asthma, cardiovascular health, cancer control, injuries prevention and control, diabetes mellitus, mental health and arthritis and musculoskeletal conditions, while the United States (US) also views heart disease and stroke, cancer and diabetes mellitus the most important health problems We need to agree what priorities should be set in Bangladesh and what targets need to be met. Before selecting the priorities of NCDs for intervention we have to obtain the population based data through surveillance.

\section{CARDIOVASCULARRISKFACTORSINSOUTHASIANS}

\section{HAM Nazmul Ahasan}

It has been predicted that by 2020, 25 million deaths worldwide (37\%) will be due to Cardiovascular disease, 6 million in developed countries and 19 million in developing countries South Asian people, comprising more than one fifth of the global population, are highly susceptible to cardiovascular diseases. Excess exposure to risk factors-the explanation that South Asians are more exposed to the causal risk factors is usually dismissed, possibly too readily. Except for smoking, which is lower in Indian (but not Pakistani and Bangladeshi) men and all South Asian women, the pattern is complex. The established risk factors com¡moner in South Asians include low high density lipoprotein cholesterol, diabetes (much commoner in South Asians), and lack of aerobic exercise. When the risk profile is seen in the context of social factors linked to coronary heart disease such as relative poverty, social upheaval after migration, and long working hours, this explanation deserves more consideration. specific risk factors, not yet established or discovered, may explain high risk. The search for a specific cause has led to many hypotheses, including the use of ghee and other cooking oils, subclinical hypothyroidism, central obesity, stress, racism, insulin resistance, a thrifty genotype, a thrifty phenotype, low vitamin C, high homocysteine, endothelial dysfunction, high levels of lipoprotein a, and other specific lipid abnormalities. No" South Asian cause” of coronary heart disease has-been proved, though each new idea has diverted attention from established risk factors. The best studied hypothesis is that the high prevalence of insulin resist;ance, independent of diabetes, underlies the high rates of coronary heart disease in South Asians. The greater prevalence of nonjinsulin dependent (type II) diabetes, impaired glucose tolerance, and insulin resistance observed in South Asian men may be important contributory factors, though South Asian men tend to have lower blood cholesterol concentrations and smoke less than white people. Studies with substantial enrolment in South Asian countries are needed to test lifestyle or pharmacologic interventions on patients who are at high risk for CVD. Programmes to promote healthy dietary patterns and physical activity in schools and workplaces should be undertaken.

\section{EVALUATION OF EFFICACYANDTOLERABILITY OF PERINDOPRIL 8MGINNEWLY DIAGNOSED STAGE-II AND UNCONTROLLED HYPERTENSIVE PATIENTS: PERINDOPRIL IN NEWLY DIAGNOSED STAGE-II \& UNCONTROLLED HYPERTENSIVE PATIENTS TRIAL (CONTROL) CONTROL STUDY GROUP}

MN Islam, M Sadequzzaman,AAS Majumder, RS Mahmud, MA Bashar, MR Ali, MAK Akanda, KQ Islae, AQM Reza, FM Siddiqui, MAAli, AK Chowdhury, MM Rahman, RC Khan, TC Ghose, J Ahmed, SK Basak, SAzam, K Pasha, TF Khan

The CONTROL (COversyl in Newly diagnosed stage-II \& unconTROlled hypertensive patients triaL) study was designed with the purpose to assess the antihypertensive efficacy and tolerability of Perindopril 8mg in newly diagnosed stage II patients and uncontrolled hypertensive patients among Bangladeshi population. This multicentre, open trial study was conducted with 245 hypertensive patients in Bangladesh. Among them 88 were newly diagnosed as stage II hypertensive patients (Group I) and 157 were patients of uncontrolled hypertension (Group II). Patients were treated with Perindopril $8 \mathrm{mg}$ for 12 weeks either in monotherapy or in addition to their existing treatment.

Male and female distribution was 57\% and 43\% respectively. Mean age of patients was 54.5 '\%11.7 years. The baseline mean sitting SBP and DBP was 163.6 '\% 17.5 mmHg and 96.8 ’\% $7.8 \mathrm{mmHg}$ respectively. 
After 12 weeks treatment, there was a significant reduction in sitting BP from baseline $(\mathrm{p}<0.001)$ in overall population as well as in Group I and Group II. In overall population, the mean sitting BP reduction was -31/-15 mmHg (from 163.6/ $96.8 \mathrm{mmHg}$ to $133.1 / 81.7 \mathrm{mmHg}$. In Group I, the reduction was -30/-18 mmHg (from 161.7/98.9 mmHg to 132.1/81.2 mmHg) and in Group II, -31/-14 mmHg (from 164.1/95.6 mmHg to $133.6 / 82 \mathrm{mmHg}$ ).

The subgroup analysis shows that the higher was the baseline SBP the greater was the reduction after 12 weeks treatment. The mean sitting BP reduction was greatest in patients with SBP > $180 \mathrm{mmHg},-42 /-15 \mathrm{mmHg}$ (from 181.4/ 97.7 mmHg to $139.8 / 83.2 \mathrm{mmHg}$ ).

Withdrawal $(4.1 \%)$ was caused by cough (2\%) and hypotension (1.2\%). Perindopril was well tolerated as indicated by the high proportion of physicians (81\%) reporting 'good' to 'excellent' tolerability at week 12.

This study suggests that, Perindopril, either as 1st line for newly diagnosed or 2nd line for uncontrolled hypertensives, is effective and well tolerated among Bangladeshi population.

\section{IN IRRITABLE BOWEL SYNDROME WHAT IS THE EFFECTIVENESS OF FIBRE, ANTISPASMODIC AND PEPPERMINTOIL?}

\section{Ahmedul Kabir}

Irritable bowel syndrome (IBS) is a functional bowel disorder characterized by abdominal pain or discomfort and altered bowel habits in the absence of detectable structural abnormalities. No clear diagnostic markers exist for IBS, thus the diagnosis of the disorder based on clinical presentation. IBS symptoms tend to come and go over time and often overlap with other functional disorder. Severity of symptoms varies and can significantly impair quality of life resulting in high health care costs. There is no single approach to treat it hence it is treated with variety of drugs and others therapies without notable enduring success. High fibre diet frequently used in treating IBS. Studies suggest that dietary fibre has multiple effects on colonic physiology. The water holding action of fibre contribute to increase stool bulk because of the ability of fibre to increase fecal output of bectaria. Fibre also speeds up colonic transit in most persons. Most investigations report increases in stool weight decrease in colonic transit time and improvement in constipation. However most studies observe no response in patient with diarrhoea or pain predominant IBS.

Clinician have observed that anticholenergic drugs may prove temporary relief for symptoms such as painful cramps related to intestinal spasm. Evidence generally supports beneficial effects of anticholenergic drugs for pain.

Peppermint oil is the major constituent of several over the counter remedies for symptoms of irritable bowel syndromes. Computerized literature searches were performed to identify all randomized controlled trials of peppermint oils for IBS. Data included medline, embase, ciscom and the cochrane library. Eight randomized controlled trails were located collectively they indicate that peppermint oil could be efficacious for symptoms relief in IBS.

\section{AREORALHYPOGLYCAEMICAGENTSEFFECTIVE IN GESTATIONALDIABETES MELLITUS(GDM)?}

\section{Rubina Yasmin}

An ever-increasing number of women with type 2 DM are going through pregnancy and with the current epidemic of obesity, more women are being diagnosed with gestational diabetes mellitus (GDM). Insulin has traditionally been the gold standard treatment in diabetic pregnancy because of its efficacy and the fact that it does not cross the placenta. How ever evidence from recent data from well designed trials and meta analysis suggest that no substantial maternal or neonatal outcome difference were found with use of oral hypoglycemic agent glyburide or metformin compared with use of insulin in women with GDM

\section{ASPIRIN IN PRIMARY \& SECONDARY PREVENTION OFVASCULAREVENTS}

\section{Md. Titu Miah}

Aspirin has long been the mainstay of treatment in both primary and secondary prevention of vascular events. Patients with moderate to high cardiovascular risk are said to benefit most with aspirin even in low doses. Indications for aspirin vary in terms of age, sex, co-morbid conditions among others. Over the years different authorities have proposed guidelines for use of aspirin. These guidelines have evolved and recommendations have changed. Recent meta-analysis has questioned the value of aspirin in primary prevention especially if it involves a significant bleeding risk. Aspirin continues to be an important part of management protocol in secondary prevention of vascular events. Patients with diabetes are twice likely to develop myocardial infarction and cerebrovascular accidents than others without diabetes. In this subgroup of patients, aspirin is less effective in primary prevention of vascular events. A common management problem facing the clinician is risk of bleeding in aspirin treated patients. Inherent to its properties, aspirin 
can cause significant morbidity by causing hemorrhage in gastrointestinal tract and central nervous system (CNS) with devastating consequences. Even then, studies have shown aspirin can prevent 14 myocardial infarction while causing 1 excess hemorrhagic stroke. With this background. the onus is on the physician to assess risk/benefit ratio and include aspirin in management of primary and secondary prevention of vascular events.

\section{DOES INTENSIVE GLUCOSE CONTROL REDUCE THE RISK OF CARDIOVASCULAR EVENTS AND MORTALITY IN PATIENTS WITHTYPE 2 DIABETES?}

\section{Shahabul Huda}

Cardiovascular complications are the major cause of death in type 2 diabetes. Several clinical studies have been carried out to see whether intensive glycemic control can reduce the cardiovascular death or not. Among them UKPDS (United Kingdom Prospective Diabetes Study), ADVANCE (Action in Diabetes \& Vascular Diseases: Preterax \& Diamicron MR Controlled Evaluation) and ACCORD (Action to Control Cardiovascular Risk in Diabetes) are considered as landmark trials. According to the UKPDS intensive glycemic control can reduce cardiovascular death, but not significantly. Then ADVANCE and ACCORD were initiated with the same objective. The type of patient and the drug classes used in these two studies were nearly same, except the use of Gliclazide MR in ADVANCE study. Both studies were able to reduce $\mathrm{HbA1c}$ level less than 6.5\%. But in case of cardiovascular death reduction the result was opposite. In the ADVANCE study cardiovascular mortality was reduced by $-12 \%$, whereas in ACCORD it was increased by $+35 \%$. According to the experts heavy use of Glimepiride and Glitazone combination and thereby frequent episodes of severe hypoglycemia and weight gain were the probable causes of increased death. Then another study VADT (Veterans Affairs Diabetes Trial) also increased cardiovascular death by using- Glimepiride and Glitazone. Taking into account these clinical studies conducted over type 2 diabetic patients it can be said that intensive glycemic control can reduce cardiovascular events and mortality. But the choice of medication and the treatment strategy need to be considered.

\section{WHATISTHE ROLEOFDIGITALISINTHETREATMENT OFHEARTFAILURE IN SINUS RHYTHM?}

\section{Anup Kumar Saha}

Is digoxin useful in patients with heart failure who remain in sinus rhythm? Generations of medical students were taught that digitalis was of little value unless the patient was in atrial fibrillation. However, the beneficial effect of digitalis in these patients was shown in several studies. This studies have shown that digitalis is useful in failure with congestion presenting in normal rhythm . Nowadays the acute positive inotropic effect of digoxin on both the failing and non-failing human heart cannot be doubted. Digoxin does have a measurable long-term positive inotropic effect on ventricular muscle. Many patients with heart failure in sinus rhythm can be managed perfectly well using modem diuretics alone. So when should digoxin be given to patients with heart failure who remain in sinus rhythm? First it should be reserved for those patients with myocardial failure. This leaves those patients whose heart failure is caused by ischaemic or primary myocardial disease whose symptoms and signs cannot be controlled with a diuretic alone; these should receive digoxin. In the remainder digoxin should be used for its inotropic effect together with a diuretic to alleviate the symptoms of fluid retention. Perhaps the future will bring us a safer alternative oral agent with a sustained positive inotropic effect, but until that time digoxin should be used in patients in sinus rhythm with myocardial failure.

\section{BETA BLOCKERS IN THE TREATMENT OF HYPERTENSION: IS IT ASUPERIOR FIRST LINE OF ANTIHYPERTENSIVE?}

\section{MdRobedAmin}

Although beta-blockers lower blood pressure in most patients, the outcomes of clinical hypertension trials of these drugs have been disappointing. No evidence exists that betablockers prevent first episodes of cardiovascular events in patients with hypertension,

and in some trials, outcomes were worse with beta-blockers than with antihypertensive drugs of other and the value of beta-blockers in treating hypertensive patients who do not have compelling indications for them has been questioned. Younger hypertensive patients have hemodynamic characteristics that would seem to be amenable to beta blocker therapy. However, most clinical trials of beta blockers did not stratify patients by age. Several meta-analyses and a few clinical trials have shown that beta-blockers may have no advantage over other antihypertensive drugs, and in fact may not reduce the risk of stroke as effectively as other classes of blood pressure medications.

Until these drugs are proved beneficial, they should be used as antihypertensive therapy only in patients with compelling cardiac indications for them or as add-on agents in those with uncontrolled or resistant hypertension 


\section{PHARMACOLOGIC MANAGEMENT OF CHRONIC NEUROPATHIC PAIN. IS IT EFFECTIVELYMANAGED ATALL?}

\section{Md. Shafiqul Bari}

Neuropathic pain (NP) affects millions of people worldwide. Many common diseases, injuries, and interventions cause NP by producing lesions in somatosensory pathways in the peripheral or central nervous system. $64 \%$ of the patients and $68 \%$ of the physicians say that NP is under treated despite presence of some guidelines. The management of patients with chronic NP is complex and response to existing treatments is often inadequate. Even with well-established NP medications, effectiveness is unpredictable, dosing can be complicated, analgesic onset is delayed, and side effects are common. Tricyclic antidepressants (TCAs) and selective serotonin and norepinephrine reuptake inhibitors (SSNRIs), calcium channel ?2-? ligands (Gabapentin and pregabalin) and Topical lidocaine are considered as first line treatment for patient with NP (grade A recommendation). Opioid analgesics and tramadol are regarded as second line therapy. Certain other antiepileptic (carbamazepine, lamotrigine, oxcarbazepine, topiramate, valproic acid) and antidepressant (bupropion, citalopram, paroxetine) medications, mexiletine, $\mathrm{N}$-methyl-D-aspartate (NMDA) receptor antagonists, and topical capsaicin are generally recommended as the third line therapy. In patients who have failed to respond to first-line medications alone and in combination, opioid analgesics or tramadol can be used as a second-line treatment alone or in combination with one of the first-line medications. Opioid analgesics and tramadol can also be considered for first-line use in select clinical circumstances. Patients who have not responded adequately to these medications used alone and in combination can be treated with one or more other recommended medications. Clinicians must consider several other factors when selecting a specific medication for a patient with NP, including: (1) the potential for adverse outcomes associated with medication-related side effects; (2) potential drug interactions; (3) comorbidities that may also be relieved by the non-analgesic effects of the medication (e.g., sleep disturbance, depression, anxiety); (4) costs associated with therapy; (5) the potential risks of medication abuse; and (6) the risks of intentional and unintentional overdose. For effective management of NP additional trials involving existing medications alone and in combination to identify characteristics of treatment responders and development of new medications are essential.

\section{GLOBAL WARMING AND EPIDEMIOLOGY OF CHOLERA-ISITCHANGING?}

\section{Fazle Rabbi Chowdhury, Chris. M. Parry}

Impact of Climate change on health is now one of the most widely discussed issue among medical scientists. Many infectious diseases now been identified as climate sensitive disease like malaria, dengue fever, tick borne diseases, cholera, leishmaniasis etc. In this presentation, changing epidemiology of cholera due to global warming will be focused. Vibrio cholerae, an important global cause of mortality and morbidity, is an autochthonous member of diverse aquatic ecosystems around the planet. It is classified according to its major surface antigen in to around 206 serogroups, of them $\mathrm{O} 1$ and $\mathrm{O} 139$ causes the disease. The World Health Organization reported in 2006 that 236,896 cases of cholera occurred in 52 countries, a $79 \%$ increase in comparison to 2005. It is now emerging in novel communities where the disease had been absent for many years, and the range of endemicity is expanding. Several distinctive features of cholera indicate a possible role of climate change in triggering its epidemic process. The bacterium thrives best in $15 \%$ salinity, 30 '\%C water temperature, and $\mathrm{pH} 8.5$. Cholera toxin and Toxin Co-regulated Pilus (TCP) are its main virulent factor which can be readily transduced into recipient strains via temperate phages. This genetic exchange allows the potential emergence of new toxigenic clones. Presence of virulence factors in environmental strains may evolve under favorable conditions (due to global warming) in to virulent strain. Increase water temperatures decrease its multiplication times and speed up growth of the organism. Sunlight influences the growth of phytoplankton which in turn raises the $\mathrm{pH}$ of the surrounding water and favors its growth. Vibrios use chitinase to play a significant role in chitin re-mineralization which can enhance its survival under starvation. Chitin also offers protection at low temperatures and under acidic conditions. Other influences under investigation include sea surface height, river discharge, and salinity, agricultural and industrial pollution etc. Poor sanitary conditions, overcrowding, improper sewage disposal and water supply, all encourage the growth and transmission of Vibrio and are in turn influenced by global warming. If the prediction of climatologists is correct and the mean temperature will rise in 1.4 to $5.8 \%$ C over the next 100 years no country will be spared. Although the doomsday scenario in the thriller film 'The Day After Tomorrow' may be far from reality, it is clear that climate is changing, challenging the health sector and the whole of human society. Cholera is just an example of that. 


\section{HYPERTENSIONISPREGNANCY}

\section{Md. Rajibul Alam}

Hypertension is pregnancy is diagnosed either from an absolute rise in blood pressure or from a relative rise above measurements obtained at booking. The conversion for the absolute value is a systolic $>140 \mathrm{mmHg}$ or a diastolic $>90$ $\mathrm{mmHg}$, the relative rise is blood pressure means either a rise in systolic pressure of $>30 \mathrm{mmHg}$ or rise in diastolic pressure of $>15 \mathrm{mmHg}$ above blood pressure at booking. There are three types of hypertension disorders of pregnancy; Chronic hypertension, gestational hypertension \& pre-eclampsia. Hypertension occurring in the second half of pregnancy in a previously normotensive woman, without significant proteinuria or other features of pre-eclampsia is termed gestational or pregnancy induced hypertension. Eclampsia is defined as occurrence of a grand mal seizure is association with pre-eclampsia.

Up to $50 \%$ of pregnancies in the DK are unplanned \& thus pre-pregnancy counseling may not be feasible. In woman with chronic hypertension, assessment before conceptions permits exclusion of secondary causes of hypertension.

Significant hypertension must be treated in it own right, regardless of the assumed underlying pathology, largely to reduce the risk or maternal intracranial hemorrhage. Treatment is mandatory when blood pressure $>170-110 \mathrm{mmHg}$. Over zealous blood pressure control may lead to placental hypotesion as placental blood flow is not auto regulated and this will compromise the fetus.

All antihypertensive drug have been shown to cross the placenta \& reach the fetal circulation. None of the antihypertensive agents in routine use have been documents to be teretogenic, although ACE inhibitors and ARBS are fototoxic. Most antihypertensive agents used in pregnancy are designated as "Category C". First line antihypertensive during pregnancy is methyldopa \& postpartum period is atenolol. A recent meta-analysis of 24 trials (2949 women) with different antihypertensive drug showed there is insufficient data to favor one agent over another, although others have concluded that agents other than parental hydralazine (Parenteral labetal or oral nifedipine) care preferable because of reduced maternal and fetal adverse effects.

\section{LIVERDISEASE INPREGNANCY}

\section{KhanAbul Kalam Azad}

Liver disease is a rare complication of pregnancy, but when it occurs it may do so in a dramatic and tragic fashion for both mother and infant. During pregnancy, the human body undergoes several changes in the process of its adaptation to the growing fetus especially liver. Although these changes are physiological, they could pose difficulty in evaluating hepatobiliary function because they may be misinterpreted as pathological. Liver is the site of many important metabolic and synthetic functions of the body. In normal pregnancy, the liver is not palpable but if it is so - it indicates definite liver disease. Due to hemodilution, biochemical tests may reveal mild increase in liver function tests.

Abnormal liver tests occur in 3\%-5\% of pregnancies, with many potential causes, including coincidental liver diseases, liver diseases induced by pregnancy and preexistent liver diseases with pregnancy. Among them pregnancy induced liver diseases typically occur at specific times during the course of pregnancy and they may lead to significant maternal and fetal morbidity and mortality. These areHyperemesis gravidarum, pre-eclamptic liver dysfunction, itrahepatic cholestasis of pregnancy(ICP), acute fatty liver of pregnancy(AFLP) and HELLP syndrome(Hemolysis, Elevated Liver enzyme, Low Platelet). There is a role for certain medications in these disorders but the risks and benefits of the use of such therapies must be considered. Treatment of these pregnancy-specific liver disorders usually involves delivery of the fetus and supportive care.

Cholestatic liver disease, autoimmune hepatitis, Wilson's disease etc may occur as coincidental diseases with pregnancy and some disease like chronic viral hepatitis may remain as pre existimg one. Management of chronic liver disease in pregnancy is directed toward optimizing control of the liver disorder whereas coincidental liver disease require therapy specific to them with some modification. Cirrhosis in the setting of pregnancy is less commonly observed but offers unique challenges for patients and practitioners. That is why management of liver disease in pregnancy requires collaboration among Internists, Obstetricians and Gastroenterologists /Hepatologist .

Liver dysfunction can appear at any point of pregnancy and causes great anxiety to the patient, her family and sometimes her medical attendants. However, when appropriately diagnosed and managed, the outcome may be favorable and the liver disease in pregnancy could resolve without any chronic consequences.

\section{ASTHMA INPREGNANCY}

\section{Mohammad Zakir Hossain}

Asthma is becoming increasingly prevalent worldwide. Several studies have investigated the effects of maternal asthma on pregnancy outcome. Overall, the literature 
suggests that asthmatic females are more at risk of low birth weight neonates, pre-term delivery and complications such as preeclampsia, especially in the absence of actively managed asthma treated with inhaled corticosteroids or asthma requiring hospitalization during pregnancy. Pregnancy with a female fetus may particularly increase the risk of these outcomes.

On the other hand, pregnancy has also effects on the course of asthma. The severity of asthma may improve, worsen or remain unchanged during pregnancy. The risk of an exacerbation requiring medical intervention may be as high as $50 \%$ in females with severe asthma and this may further increase the risk of poor outcomes, particularly low birth weight and pre-term delivery. Nevertheless, observational data showing strong associations between poor asthma control during pregnancy (as evidenced by symptoms, impaired pulmonary function, or exacerbations) and these increased risks suggest that better asthma control may improve pregnancy outcomes. Treatment also may reduce serious risks to the mother resulting from uncontrolled asthma, including death. However, the choice among medications must take into account their potential adverse effects on the fetus.

Asthma treatment, by reducing maternal inflammation and preventing exacerbations contributes to improved outcomes for both mother and fetus.

\section{DEEP FUNGAL INFECTIONS - AN EMERGING PROBLEM INBANGLADESH}

\section{Rukhsana Parvin, Muntasir Hasnain, Khan Md Arif, Md Shahriar Mahbub, Md Robed Amin, Md Titu Miah , Ratan Das Gupta, Md Billal Alam, HAM Nazmul Ahasan}

Objective: Invasive deep fungal infections have become a major cause of morbidity and mortality over the past three decades. Organ transplantation, the use of aggressive chemotherapy and the availability and widespread use of immunosuppressive treatments for many medical ailments have resulted in large populations of patients who are at risk of fungal infections. We report our experience to increase awareness of the clinical spectrum of disseminated fungal infection and its similarity to other infections and malignancy.

Materials and Methods: Case reports from different medical institution for last 6 months were searched through case registry and recent scientific presentation. Original article and case series were searched for disseminated fungal infection in Bangladesh from all available national and international journal. The search item was Deep,
Disseminated, Fungal, Histoplasmosis, Aspergillosis, Mucormycosis, Blastomycosis etc. Search engine was google. Pubmed and Cochrane library were also searched for case reports of disseminated fungal infection from Bangladedsh.

Results: Recently a case of Histoplasmosis was reported in Dhaka Medical College Hospital (DMCH) where a 57 years old male presented with low grade fever for 3 months, backache for 2 and a half months followed by progressive weakness of both lower limbs and constipation along with incontinence of urine for the same duration. On examination, there was spastic paraplegia with sensory loss upto the level of D10. Radiological evidence of X-ray and Magnetic Resonance Imaging(MRI) were reduction of intervertebral space, destruction of vertebral body with a paravertabral soft tissue mass mimicking features suggesting of Tuberculosis or Malignancy. An open biopsy from paravertebral soft tissue showed histopathological features consistent with Histoplasmosis. Other deep fungal infections eg Pulmonary Blastomycoses, Mucormycoses and Pulmonary Aspergilloma were also reported in DMCH and Bangobandhu Shiekh Mujib Medical University(BSMMU).

Conclusion: As these fungal infections are not commonly encountered in our country and most of the dissemination has similar clinical features of chronic inflammatory process and malignancy, we need a high index of suspicion to diagnose with different diagnostic approach. Bangladesh may be experiencing increase load of disseminated fungal infections and awareness is now very important to explore its underlying aetiology.

\section{REACTIVATION OF HEPATITIS B VIRUS(HBV) FOLLOWING CANCER CHEMOTHERAPY-A CASE REPORT}

\section{Md Moklesur Rahman}

Objective: Reactivation of HBV may occur after immunosuppressive and cancer chemotherapy. some of these cases may develop liver failure in a previously compensated disease. Aim of this case report is to make awareness of this reactivation in our society after cancer chemotherapy or immunosuppressive therapy.

Methods and Results: A 45 year old lady,mother of one child was found to have positive HBsAg since 1994 when the test was done for vaccination. She was asymtomatic and clinically unremarkanble. she developed carcinoma of left breast which was operated in July 2008. Combination cancer chemotherapy 
was started. After 5 th cycle of chemotherapy she developed rise of ALT and HBV DNA. Patient was treated with tab lamivudine $100 \mathrm{mg} /$ day. After one year of follow up she had no symptoms and ALT and HBV DNA became normal.

Conclusion: Reactivation of HBV DNA may occur after cancer chemotherapy or immunosuppressive therapy. At present recommendation of APASL,EASL and AASLD is to start prophylactic Lamivudine, Entecavir or any other antiviral drugs in all patients who are positive for HbsAg irrespective of HBeAg, ALT or HBV DNA status before starting chemotherapy or immunosuppressive therapy. So, HbsAg should be tested in all such cases.

\section{SEROLOGICALCO-RELATIONWITHDIAGNOSISAND BIOCHEMICALCO-RELATIONWITHSEVERITY IN 86 ADMITTEDDENGUECASES}

\section{K. Nazimuddin, RSC Sarker, A.K.M Shaheen, M Rahman, WMMHaque}

Objectives: This prospective study was designed to evaluate the correlation between biochemical changes and severity of dengue infection and serological relation in diagnosis.

Method: A study was carried out at BIRDEM from January, 2006 to December, 2009. A total of 86 dengue patients were recruited. Blood sample of Fasting Sugar, 2HABF, S. creatinine, SGOT, SGPT, LDH, S. Calcium, platelet count, anti Dengue IgM and anti Dengue IgG with antibody Index were done.

Results: Total 86 cases of clinically and serologically diagnosed dengue infection were classified into 4 groups, i.e. 30 cases of dengue fever, 33 cases of Dengue Haemorrhagic Fever (DHF-I), 15 of DHF-II and 8 were in DHF-III catagory. Among 86 patients 53(61.6\%) were male \& 33(38.4\%) were female. The study showed that both the levels of SGOT and SGPT were significantly higher. SGPT were above normal cutoff value $40 \mathrm{IU}$ in $59.3 \%$ cases and in case of SGOT $65.7 \%$ are above cutoff value. S. creatinine was above normal in just $4.3 \%$ cases and not proportionate to the finding of liver enzymes. Surprisingly S. bilirubin was normal in most cases with mean of $0.82 \mathrm{mg} / \mathrm{dl}$ ('\%0.48). Serum calcium was proportionately lower with severity. Fasting and ABF did not co-relate with severity. Platelet count co-relates well with severity with mean of $25000 / \mathrm{cmm}$ in DHF-III and 28000 in DHF-II. Anti dengue IgM and IgG were done in all cases and 58(49.9\%) were IgM positive and 58(67.4\%) were positive for IgG. Both IgM and IgG were positive in 25 cases. More than 37\% of total diagnosed DHF patients were IgM negative.
Conclusion: SGOT and SGPT both are increased in dengue patients but SGOT was more increased than SGPT. Serum calcium was found lower in DF. Low platelet count correspond well with severity of Dengue infection. Antibodies, both the IgM and IgG poorly co-relates with diagnosis.

\section{STUDY OF NON-TRADITIONAL CARDIOVASCULAR RISK FACTORS IN CHRONIC KIDNEY DISEASE (CKD) ANDHAEMODIALYSISDEPENDENTPATIENTS-ACASE CONTROLSTUDY}

\section{Ratan Das Gupta}

Objective: Cardiovascular disease leading cause of mortality and morbidity in CKD and haemodialysis dependent patients. Mortality rates are 10-20 times higher among patients with end stage renal disease, compared with general population.This excess risk is not entirely explained by elevation of traditional risk factors. The study was conducted to see elevation of Several Non-traditional risk factors associated with an increased risk for cardiovascular disease in CKD and haemodialysis dependent patients.

Materials and Methods: 48 non-dialysis CKD, 22 Heamodialysis dependent patients and 26 healthy controls were included in the study. Cases were selected define inclusion and exclusion criteria and age, sex match with control. All case was investigated for cardiovascular disease and non-traditional risk factor (homocysteine, fibrinogen, CRP, factor VII activity and haemoglobin). All results presented as mean, percentage and to compared with control ANOVA and chi-square and t -test applied.

Results: Mean Homocysteine 15.38 \% 5.06, 27.30 '\% 31.12, 23.76 \% 9.15 a\%mol/L in control, non-dialysis CKD and haemodialysis dependent patient respectively. Fibrinogen in control, non-dialysis CKD and haemodialysis dependent patient were , 180.25 ’\% 40.64, 264.10 ’\% 67.81, 259.59 ’\% $60.92 \mathrm{mg} / \mathrm{dl}$ respectively. CRP was in control, non-dialysis CKD and haemodialysis dependent patient were 3.90\% 1.03 52.59 \% $\%$ 82.16, 17.31 \% 18.42 mg/L respectively. Factor VII activity in control94.18 '\% 12.66, non-dialysis CKD 103.97 '\% 14.41, haemodialysis dependent patient 106.18 '\% 14.64 . Haemoglobin was in control $13.85 \%$ 1.59gm/dl, non-dialysis CKD $13.85 \% 1.59$ gm/dl, Haemodialysis dependent patients9.46 $\% 1.87 \mathrm{gm} / \mathrm{dl}$.cardiovascular disease in nondialysis CKD 54.56\% and haemodialysis dependent patients 59.4\%. In compared with control both non-dialysis CKD and haemodialysis dependent patients homocysteine, fibrinogen , CRP, factor VII significantly increased and haemoglobin was significantly low. Prevalence of cardiovascular disease 
increased in both non-dialysis CKD and haemodialysis dependent patients.

Conclusion: Non-traditional cardiovascular risk factors were significantly increased along with traditional common traditional cardiovascular risk factors in CKD and haemodialysis dependent patients.

\section{PREVALENCE OF CRYPTOGENIC CIRRHOSISAMONG ADMITTED HEPATIC CIRRHOSIS PATIENTS}

\section{Khan Fariduddin Mohammad Ayaz, HAM Nazmul Ahasan}

Objective: Cryptogenic cirrhosis is a diagnosis of exclusion. When the etiology of cirrhosis remains obscure it is labeled as cryptogenic. This study was carried out in Dhaka Medical College Hospital with the objective to find out the prevalence of cryptogenic cirrhosis among admitted cirrhosis patients.

Material and Method: Diagnosis of cirrhosis was done by features of chronic hepatic insufficiency and portal hypertension by both clinical and laboratory measurement. Liver biopsy was done in selective cases. All patients were screened for Hepatitis B and Hepatitis C virus. Suspected cases were investigated for wilsons disease and alcoholism etc.

Results: Out of 131 cases included in the study 17 (13.0\%) were cryptogenic and the most common cause of cirrhosis was Hepatitis B (55.0\%) followed by Hepatitis C (17.6\%), alcoholic (3.8\%) and Wilson's (3.1\%). Cryptogenic cirrhosis was almost consistent in male and female which were $12.5 \%$ and $14.3 \%$ respectively. But the mean age of the female patients were significantly $(\mathrm{p}=0.024)$ higher than the male. Weight loss was significantly $(\mathrm{p}=0.05)$ less among the cryptogenic patients with the female patients having no weight loss at all. Out of the five female cryptogenic cases four had diabetes mellitus. There was a significantly less rise in Prothrombin Time (PT) among the cryptogenic patients and none of the cryptogenic cases had Space Occupying Lesion (SOL). Occult viral ingections, NASH, ?-1 Antitrypsin deficiency and non $\mathrm{B}$ non $\mathrm{C}$ virus like $\mathrm{G}$ virus may be the cause of these cryptogenic cases. '

Conclusion: Identifying the cause behind cirrhosis at times may require extensive investigation facilities as a result the prevalence may vary from country to country. Gradual improvement in the diagnostic techniques and advancement in laboratory facilities will soon make a way for finding out the etiology in all the cases.
ASSOCIATION BETWEENSTROKEAND METABOLIC SYNDROME:ANOBSERVATIONALSTUDY

Md. Jabed Iqbal, Md. Mahmudur Rahman Siddiqui, Ekramul Mustafa, Ahmed Hossain, Quazi Tarikul Islam

Objective: Metabolic syndrome is the cluster of vascular risk factors includes: insulin resistance, elevated blood pressure, hyperlipidemia, and obesity. Patient with metabolic syndrome is strongly associated with cardiovascular and cerebrovascular disease. The aim of this study is to find out association between stroke and metabolic syndrome in our population.

Methods: In this observational study, definition of metabolic syndrome was taken from the guidelines by the National Cholesterol Education Program Adult Treatment Panel III. This is an observational type study. Randomly selected, a total number of fifty patients were studied from July 2009 to December 2009 at Medicine ward of Dhaka Medical College Hospital.

Results: Among the study population $46 \%$ had metabolic syndrome which were more prevalent among men than women ( $48 \%$ of men vs $20 \%$ of women). This study estimated that metabolic syndrome may accounts for about 58\% ischemic stroke. Only $4 \%$ patient was found obese. With or without metabolic syndrome most of the patient had elevated TG (64\%) and hypertension (64\%).

Conclusion: Without obesity, metabolic syndrome is an important risk factor for cerebrovascular disease. Majority of the patient with metabolic syndrome presents as ischemic stroke.

\section{EVALUATION OF ADVERSE EFFECTS OF SODIUM STIBOGLUCONATE INTHETREATMENTOFVISCERAL LEISHMANIASIS(KALA-AZAR)}

Md. Abdus Sattar Miah, A R M Saifuddin Ekram, Md. Azizul Hoque, Md. Abdus Salam, A B M Saiful Alam, Md. Zahirul Haque, AK M Rafiqueuddin

Objective: Sodium Stibogluconate is still used in the treatment of visceral leismaniasis in Bangladesh. Despite its major and minor adverse affect, it is still a common drug for elimination of Kala azar in our country.Systemic observation of adverse event of this drug was not done before.

Material and Methods: This descriptive cross sectional study was carried out on 30 cases of visceral leishmaniasis in the Department of Medicine, Rajshahi Medical College Hospital to evaluate the adverse effects of sodium stibogluconate in the treatment of visceral leishmaniasis. 
Results: Out of 30 patients, 19 patients (63.33\%) developed abnormalities in ECG, among them 14 patients (46.67\%) developed prolonged QTc, 6 patients (20\%) developed Twave inversion and 1 patient (3.33\%) developed transient first degree heart block. No patient developed symptomatic arrhythmia. Five patients (16.67\%) developed transient raise of bilirubin, 11 patients (36.67\%) developed raised SGPT. None of them developed clinical hepatitis. 23 patients (76.67\%) developed raised serum amylase but none developed clinical pancreatitis. There was no change in renal function. No adverse effects were noted on complete blood count.

Conclusion: Sodium stibogluconate can be used safely in Kala-azar patients with adequate monitoring. Identification of factors before and during treatment that may increase the risk of QTc prolongation and arrhythmia is important for prevention of deadly complications.

\section{DEPRESSION IN PATIENTS WITH RHEUMATOID ARTHRITIS INATEACHINGHOSPITAL}

Md. MahbubulAlam, ARM Saifuddin Ekram, MAMamun Hussain, Md.Azizul Haque,D Hasan Tarik, Md. Mahbubur Rahman Khan , Md. Zakir Hossain

Objectives: This cross-sectional comparative study was carried out in the Department of Medicine, Rajshahi Medical College Hospital to evaluate the association between rheumatoid arthritis and depression.

Material and Method: A total of 61 patients were selected, RA group comprising of 30 patients and Non RA healthy group comprising of 31 patients. Diagnosis of rheumatoid arthritis was based on ARA criteria and diagnosis of depression was carried out by "Criteria for depressive episode” in ICD 10 and severity (grading) of depression was assessed by the Hamilton Rating Scale for Depression.

Results: Among the RA group (30 patients) 7 (23.3\%) persons were found to have depressive illness while 3 (10\%) patients had depression as symptoms, 5 (16.7\%) had mild depression and 2 (6.7\%) had moderate depression. None suffered from severe depression. Among the healthy (control) group 1 (3.2\%) person had mild depression.
Conclusion: Depression is increased significantly in patients with RA compared to non-RA controls. We feel that a greater emphasis on recognizing and treating depression in RA is warranted.

\section{RIGHT VENTRICULAR MYOCARDIAL INFARCTION: PRESENTATION AND OUTCOME IN A TEACHING HOSPITAL}

Md. Nazmul Huda, A R M Saifuddin Ekram, Md. Zahidus Sayeed, Iftekhar Mahmood, Khalilur Rahman, Mir Mahfuzul Hoque Chowdhury, MA Jalil Chowdhury

Objective: Right Ventricular Infarction (RVI) complicating inferior wall myocardial infarction (MI) is common, associated with morbidity and mortality. We try to systematically assess the frequency, clinical presentation and in hospital outcomes of right ventricular myocardial infarction in a tertiary-care set up.

Material and Method: This study was a descriptive, cross sectional observational series of consecutive patients with RVMI. All patients with acute inferior myocardial infarction ( $n=100$ ) were enlisted. RVMI was diagnosed by ?1mm ST elevation in lead V4R in right sided electrocardiogram. Right ventricular myocardial infarction (RVI) occurred in 31\% ( $\mathrm{n}=31$ ) of patients of acute inferior infarctions. Patients with isolated inferior myocardial infarction served as controls $(n=69)$. Echocardiography was performed within 24 hours of admission. From both groups, 51\% were qualified for thrombolysis

Results:. The incidence of hypotension (96.7\%), cardiogenic shock (64.5\%) and bradycardia and heart block were much higher in RVI than in inferior myocardial infarction. Clinically manifest RV dysfunction (raised jugular venous pulse, hypotension and tricuspid regurgitation) and right ventricular dilatation detected by echocardiography was seen in a variable number of patients. The in hospital mortality rate was significantly higher $(n=13,41.9 \%)$ in right ventricular infarction group than in inferior myocardial infarction group $(n=2,2.9 \%)$.

Conclusion: Right ventricular infarction is a distinct, easily diagnosable entity occurring frequently in the setting of AIMI. Early recognition, close monitoring and early PCI may be beneficial and reverse the vicious cycle leading to cardiogenic shock and death due to RVMI. 